\title{
Mediastinal Lymphadenopathy in Visceral Leishmaniasis
}

\author{
Surinder Singh Rana ${ }^{1}$ Nalini Gupta ${ }^{2}$ Ravi Sharma ${ }^{1}$ Pankaj Kumar ${ }^{2}$ \\ ${ }^{1}$ Department of Gastroenterology, Post Graduate Institute of Medical \\ Education and Research (PGIMER), Chandigarh, India \\ 2 Department of Cytology and Gynecological Pathology, Post \\ Graduate Institute of Medical Education and Research (PGIMER), \\ Chandigarh, India \\ Address for correspondence Surinder Singh Rana, MD, DM, \\ Department of Gastroenterology, Postgraduate Institute of Medical \\ Education and Research (PGIMER), Chandigarh 160012, India \\ (e-mail: drsurinderrana@yahoo.co.in).
}

\author{
Abstract \\ Keywords \\ - endosonography \\ - tuberculosis \\ - kala-azar \\ - lymph nodes \\ - fine needle aspiration
}

Visceral leishmaniasis (VL) usually presents with malaise, fever, weight loss, and hepatosplenomegaly. Lymphadenopathy is rarely reported in patients with VL from India. We report an interesting case of VL, presenting with malaise, fever and weight loss along with isolated mediastinal lymphadenopathy and mimicked tuberculosis. Absence of spleen because of previous splenectomy for symptomatic portal hypertension led to difficulty in establishing the correct diagnosis that was eventually established by endoscopic ultrasound (EUS)-guided fine-needle aspiration (FNA).

\section{Introduction}

Visceral leishmaniasis (VL) or Kala-azar is a disseminated protozoan infection that spreads via the bite of phlebotome sand flies and is caused by Leishmania sp. ${ }^{1}$ The disease onset is usually insidious and typical manifestation of VL include slow progression of malaise, fever, weight loss, and hepatosplenomegaly. ${ }^{1}$ Kala-azar (â€œblack feverâ€) refers to the dark color of skin seen in patients with VL and is usually seen in patients in South Asia but not elsewhere. ${ }^{2}$ Lymphadenopathy is usually reported in cases of VL from East African countries and is rarely reported from India. ${ }^{3} \mathrm{VL}$ is usually diagnosed by demonstrating parasites in the bone marrow or splenic aspirate, and fine-needle aspiration (FNA) from enlarged lymph nodes has been occasionally reported for its correct diagnosis. ${ }^{1,3}$ We report an interesting case of $\mathrm{VL}$ presenting with malaise, fever and weight loss along with isolated mediastinal lymphadenopathy and mimicked tuber- culosis. Absence of spleen because of previous splenectomy for symptomatic portal hypertension led to difficulty in establishing the correct diagnosis that was eventually established by endoscopic ultrasound (EUS)-guided FNA.

\section{Case Report}

A 34-year-old male, who had undergone splenectomy with proximal lienorenal shunt 12 âêyears ago for variceal bleeding because of extrahepatic portal venous obstruction, was admitted with a 2-month history of fever, fatigue, and weight loss. The general physical examination revealed a thin built patient with no organomegaly or lymphadenopathy. The hematological investigations revealed hemoglobin of 8.2 $\mathrm{g} / \mathrm{dL}$, total leukocyte count of 5,200 cells//1 $1 / 4 \mathrm{~L}$, and platelet count of $1.3 \mathrm{lac} / \hat{1} 1 / 4 \mathrm{~L}$. Blood cultures as well as tests for human immunodeficiency virus (HIV) were negative. Chest X-ray did not reveal any abnormal findings. Liver function tests
DOI https://doi.org/ 10.1055/s-0040-1715983. ISSN 0976-5042. (c) 2022. Society of Gastrointestinal Endoscopy of India. All rights reserved.

This is an open access article published by Thieme under the terms of the Creative Commons Attribution-NonDerivative-NonCommercial-License, permitting copying and reproduction so long as the original work is given appropriate credit. Contents may not be used for commercial purposes, or adapted, remixed, transformed or built upon. (https://creativecommons.org/ licenses/by-nc-nd/4.0/)

Thieme Medical and Scientific Publishers Pvt. Ltd., A-12, 2nd Floor, Sector 2, Noida-201301 UP, India 


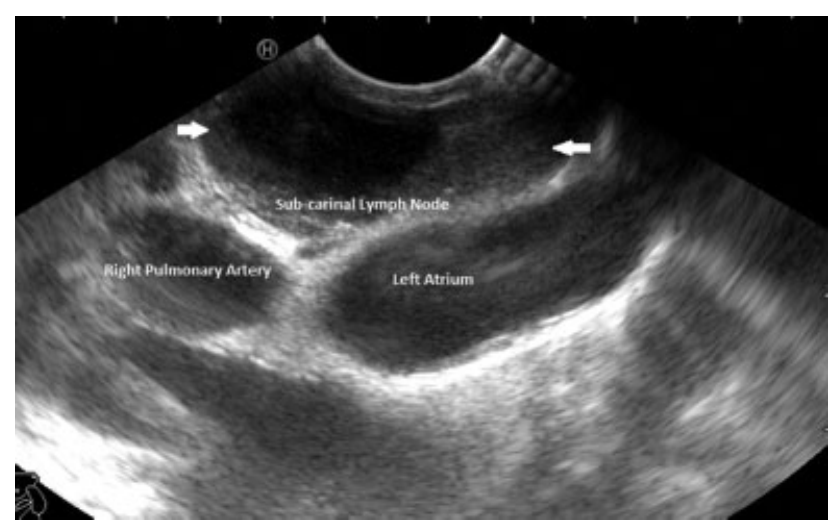

Fig. 1 Endoscopic ultrasound (EUS): enlarged hypoechoic subcarinal lymph node with areas of necrosis.

revealed conjugated hyperbilirubinemia (total/conjugated: $2.5 / 1.9 \mathrm{mg} / \mathrm{dL})$, elevated alkaline phosphatase $(3,326 \mathrm{U} / \mathrm{L}$; normal: $<128 \mathrm{U} / \mathrm{L}$ ), low-serum albumin $(2.8 \mathrm{~g} / \mathrm{dL}$; normal $3.4 a ̂ €$ “ $4.8 \mathrm{~g} / \mathrm{dL}$ ), and elevated serum globulins $(4.6 \mathrm{~g} / \mathrm{dL}$; normal 2.0â€“3.5 g/dL). Abdominal ultrasonography revealed dilated intrahepatic biliary radicles due to portal cavernoma cholangiopathy (PCC). There were no gastroesophageal varices on gastroscopy.

EUS done for evaluation of PCC revealed enlarged hypoechoic subcarinal as well as aortopulmonary lymph nodes with areas of necrosis ( - Fig. 1, arrows). EUS-guided FNA was performed using 22G EUS FNA needle (EchoTip, Wilson-cook Medical, Winston-Salem, North Carolina, United States) (-Fig. 2), and cytological examination of lymph node aspirate revealed collection of foamy histiocytes, showing numerous amastigote forms of Leishmania donovani (-Fig. 3: MGG x40). Higher magnification revealed multinucleated histiocyte, showing Leishmania donovani (LD) bodies ( - Fig. 4) and safety pin-shaped LD bodies with kinetoplasts within a histiocyte (-Fig. 5; arrows [MGG x100]). RK 39 serological test was positive. Patient was successfully treated with liposomal amphotericin B.

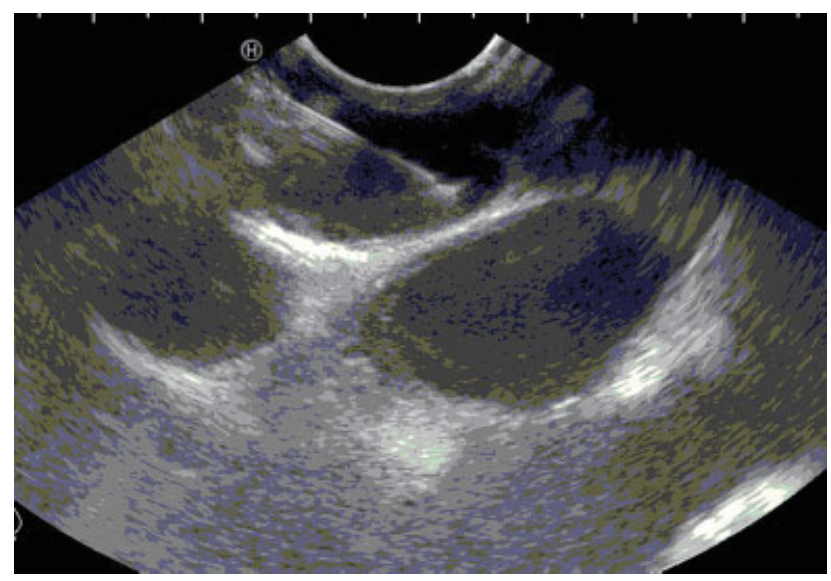

Fig. 2 Endoscopic ultrasound (EUS)-guided fine-needle aspiration (FNA) was performed using 22G EUS FNA needle.

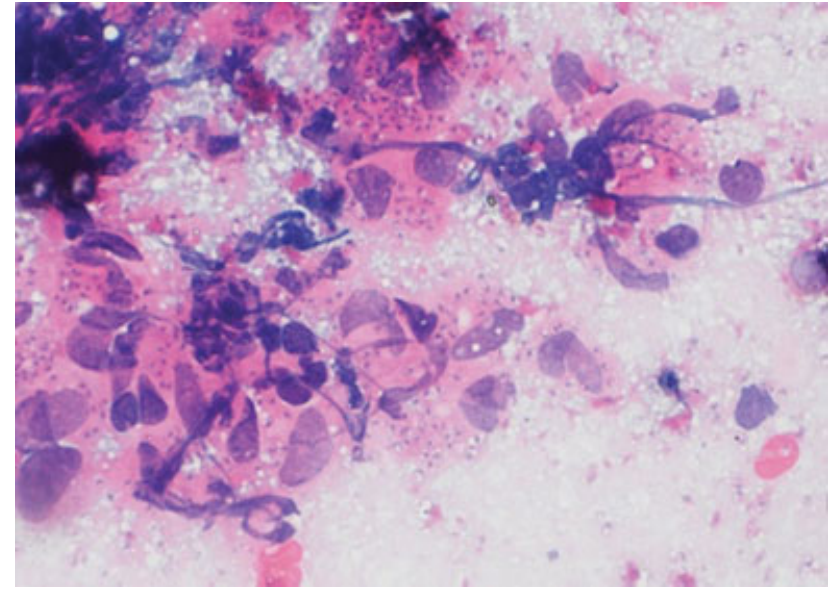

Fig. 3 Cytological examination of lymph node aspirate revealed collection of foamy histiocytes, showing numerous amastigote forms of Leishmania donovani (MGG $\times 40$ ).

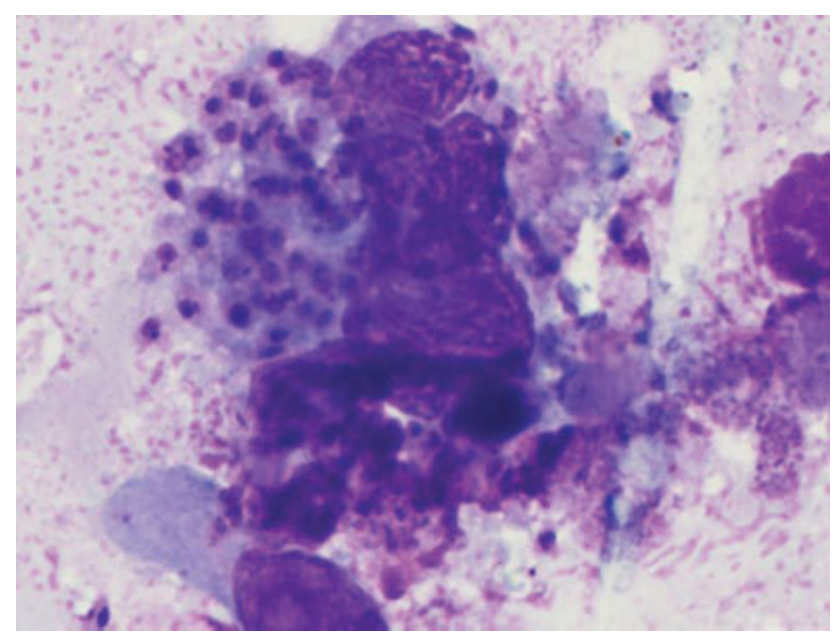

Fig. 4 Multinucleated histiocyte showing Leishmania donovani (LD) bodies (MGG $\times 100)$.

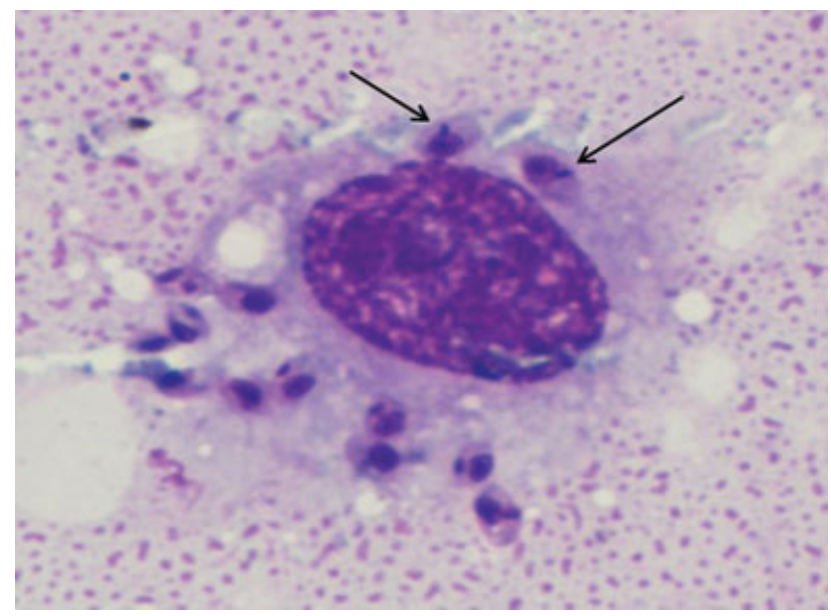

Fig. 5 Safety pin-shaped Leishmania donovani (LD) bodies with kinetoplasts within a histiocyte (arrows [MGG ×100]). 


\section{Discussion}

Patients with VL in India usually present with fever, weight loss, hepatomegaly, splenomegaly, pancytopenia, and hyperglobulinemia. Enlarged firm spleen in patient with febrile illness from an endemic area raises suspicion of VL. Our patient had undergone splenectomy for symptomatic extrahepatic portal venous obstruction and did not reside in the endemic area for VL. Therefore, Kala-azar was not considered as a differential diagnosis in the index case. Also, the clinical presentation as well as EUS morphology of mediastinal lymph nodes mimicked lymph nodal tuberculosis., ${ }^{4,5}$

Lymphadenopathy due to Kala-azar is rare in India and isolated mediastinal lymphadenopathy in the absence of HIV infection is very rarely reported in $\mathrm{VL} .{ }^{6,7} \mathrm{VL}$ is commonly diagnosed by demonstrating parasites in the bone marrow and splenic aspirate and lymph node FNA has been rarely used for its diagnosis. ${ }^{1-3}$ Most of the cases of lymph nodal leishmaniasis have been diagnosed by aspiration of cervical lymph nodes or ultrasound-guided FNA of mesenteric lymph nodes. ${ }^{1-3}$ The diagnosis of VL by EUS-guided FNA of mediastinal lymph nodes is rarely reported in the literature. ${ }^{6,7}$

In conclusion, leishmaniasis should be included in differential diagnosis of unexplained lymphadenopathy, especially in endemic areas. EUS-guided FNA is an useful modality for correctly diagnosing unexplained mediastinal lymphadenopathy.

Funding

No funding sources.

\section{Conflicts of Interest}

There are no conflicts of interest and no financial disclosures to be made.

\section{References}

1 van Griensven J, Diro E. Visceral leishmaniasis: recent advances in diagnostics and treatment regimens. Infect Dis Clin North Am 2019;33(01):79-99

2 UpToDate. Visceral leishmaniasis: clinical manifestations and diagnosis. Available at: https://www.uptodate.com/contents/visceralleishmaniasis-clinical-manifestations-and-diagnosis?search=kala $\% 20$ azar\&source=search_result\&selectedTitle $=1 \sim 82 \&$ usage_type $=$ default\&display_rank=1. Accessed May 23, 2020

3 Agarwal P, Kumar V, Kaushal M, Kumari M, Chaudhary A. Indian visceral leishmaniasis with extensive lymphadenopathy - An unusual presentation: A case report with literature review. Cytojournal 2017;14:9

4 Rana SS, Bhasin DK, Singh K. Endoscopic ultrasound features of mediastinal tuberculosis. Endoscopy 2012;44(01):106-107, author reply 107

5 Rana SS, Bhasin DK, Srinivasan R, Singh K. Endoscopic ultrasound (EUS) features of mediastinal tubercular lymphadenopathy. Hepatogastroenterology 2011;58(107-108)819-823

6 Choudhary NS, Kataria S, Guleria M, Puri R. Leishmaniasis presenting as small isolated mediastinal lymphadenopathy diagnosed by endoscopic ultrasound-guided fine-needle aspiration. Endoscopy 2015;47(Suppl 1 UCTN)E147-E148

7 Sihuay D, Gornals JB, Saumoy Met al.Visceral leishmaniasis with mediastinal lymphadenopathy diagnosed with endoscopic ultrasound-guided fine needle aspiration. Rev Esp Enferm Dig 2016; 108(11):736-738 